\title{
Kalman interpolation filter for channel estimation of LTE downlink in high-mobility environments
}

\author{
Xuewu Dai ${ }^{1}$, Wuxiong Zhang ${ }^{2,3,4}$, Jing $\mathrm{Xu}^{3,4}$, John E Mitchell ${ }^{1 *}$ and Yang Yang ${ }^{3,4}$
}

\begin{abstract}
The estimation of fast-fading LTE downlink channels in high-speed applications of LTE advanced is investigated in this article. In order to adequately track the fast time-varying channel response, an adaptive channel estimation and interpolation algorithm is essential. In this article, the multi-path fast-fading channel is modelled as a tapped-delay, discrete, finite impulse response filter, and the time-correlation of the channel taps is modelled as an autoregressive (AR) process. Using this AR time-correlation, we develop an extended Kalman filter to jointly estimate the complex-valued channel frequency response and the AR parameters from the transmission of known pilot symbols. Furthermore, the channel estimates at the known pilot symbols are interpolated to the unknown data symbols by using the estimated time-correlation. This article integrates both channel estimation at pilot symbols and interpolation at data symbol into the proposed Kalman interpolation filter. The bit error rate performance of our new channel estimation scheme is demonstrated via simulation examples for LTE and fast-fading channels in high-speed applications.
\end{abstract}

Keywords: LTE advanced, Channel estimation, Extended Kalman filter, Pilot-aided-interpolation

\section{Introduction}

Channel estimation plays an important role in communication systems and, particularly, in the 3GPP Long-Term Evolution (LTE) which aims at continuing the competitiveness of the 3G Universal Mobile Telecommunications System technology. Orthogonal frequency-division Multiple Access (OFDM) is considered as one of the key technologies for the 3GPP LTE to improve the communication quality and capacity of mobile communication system. As the support of high mobility is required in 3GPP LTE systems, the signals at the OFDM receivers are likely to encounter a multi-path, fast time-varying channel environment [1]. Thus, good channel estimation and equalization at the receiver is demanded before the coherent demodulation of the OFDM symbols. In mobile communication, since the radio channel is modelled by some dominant spare paths and is represented by path taps, the channel estimation is to estimate and track the channel taps adaptively and efficiently.

In wideband mobile communications, the pilot-based signal correction scheme has been proven a feasible

\footnotetext{
* Correspondence: j.mitchell@ee.ucl.ac.uk

${ }^{1}$ Department of Electronic \& Electrical Engineering, University College

London, Torrington Place, London WC1E 7JE, UK

Full list of author information is available at the end of the article
}

method for OFDM systems. The 3GPP LTE standard employs a Pilot Symbol-Aided Modulation (PSAM) scheme but does not specify the methods for estimating the channel from the received pilot and data signals. In the 3GPP LTE downlink, pilot symbols, known by both the sender and receiver, are sparsely inserted into the streams of data symbols at pre-specified locations. Hence, the receiver is able to estimate the whole channel response for each OFDM symbol given the observations at the pilot locations. Pilot-symbol-aided channel estimation has been studied [2-4] and the common channel estimation techniques are based on least squares (LS) or linear minimum mean square error (LMMSE) estimation [5]. Note that most pilot-symbol-aided channel estimators, including those mentioned above, work in the frequency domain. LS estimation is the simpler algorithm of the two as it does not use channel correlation information. The LMMSE algorithm makes use of the correlation between subcarriers and channel statistic information to find an optimal estimate in the sense of the minimum mean square error.

In the literature, based on these two basic estimators, various methods are proposed to improve the performance of the channel estimation. As the LS and LMMSE estimators only give the channel estimate at the pilot 
symbol, most current work on pilot-aided channel estimation considers interpolation filters where channel estimates at known pilot symbols are interpolated to give channel estimates at the unknown data symbols. Since the 3GPP LTE downlink pilot symbols are inserted in a comb pattern in both the time and the frequency domain, the interpolation is a $2 \mathrm{D}$ operation. Although some 2D interpolation filters have been proposed [6], presently, interpolation with two cascaded orthogonal 1D filters is preferred in 3GPP LTE. This is because the separation of filtering in time and frequency domains by using two 1D interpolation filters is a good trade-off between complexity and performance. Various 1D interpolation filters have been investigated. Examples are linear interpolation, polynomial interpolation [7], DFT-based interpolation [8], moving window [9] and iterative Wiener filter [10].

From a system point of view, the channel estimation is a state estimation problem, in which the channel is regarded as a dynamic system and the path taps to be estimated are the state of the channel. It is known that Kalman filter (KF) provides the minimum mean square error estimate of the state variables of a linear dynamic system subject to additive Gaussian observation noise [11]. By considering the radio channel as a dynamic process with the path taps as its states, the KF has shown its suitability for channel estimation in the time domain [1]. In the frequency domain, Kalman-based channel estimator in OFDM communication has also been studied $[1,12,13]$. For example, in $[1,12]$, a modified KF is proposed for OFDM channel estimation where the time-varying channel is modelled as an autoregressive(AR) process and the parameters of the AR process are assumed real and within the range $[0.98,1]$ for slow-fading channels. However, in the high-mobility environment, these parameters are relative large (e.g. in the $200 \mathrm{~km} / \mathrm{h}$ environment, they are complex values with magnitudes varying in $[0,1.5])$ representing a fastfading channels.

The difference between the KF in [12] and the one proposed in this article is that the former estimated the parameters of AR by a gradient-based recursive method separately, rather by the linear KF. Whereas, we derive an extended Kalman filter (EKF) for jointly estimating the channel response and the parameters of the AR model simultaneously. In addition, the parameters of the AR model are assumed time-invariant and known in priori by solving Yule-Walker equation in [1]. The authors of [13] only considered the comb-type pilot patterns in which some subcarriers are full of pilot symbols without unknown data. As a result, the KF in [13] requires continuous stream of pilot symbols and is not suitable for 3GPP LTE, as the 3GPP LTE employs a scattered pattern where the pilot symbols are distributed sparsely among the data streams.
Although the KF-based channel estimation for LTE uplink has been reported recently [1], there has been no KF-based joint estimation of both time-varying channel taps and the time-correlation coefficients of 3GPP LTE downlink in frequency-time domain. This article focuses on the major challenge of scattered pilot-aided channel estimation and interpolation for a time-varying multipath fast-fading channel in 3GPP LTE downlink. An AR process is used to model the time-varying channel. Both the taps of the multipath and the timecorrelation coefficients are jointly estimated by treating the channel as a nonlinear system. Then, a combined estimation and interpolation scheme is present under the EKF framework.

The main contribution of the proposed method is (1) both the time-correlation coefficients and channel taps are estimated simultaneously in the framework of EKF; (2) no assumption on the upper/lower boundaries of the time-correlation coefficients to achieve a good tracking of fast-fading channel in high-mobility scenario; (3) applicable to preamble pilot patterns, comb-type pilot patterns and scattered pilot patterns.

This article is organized as follows: Section "System model" gives an overview of the LTE 3GPP downlink system and formulates its channel estimation problem. In Section "EKF for channel estimation", an EKF is derived by using a first-order Taylor approximation for the joint estimation of channel taps and time-correlation coefficients at pilot symbols. Section "EKF for channel interpolation" describes the combined estimation and interpolation scheme and summarizes the proposed algorithm. Simulation results of the proposed Kalman interpolation filter are presented and its performance is demonstrated in Section "Simulation results and performance analysis".

\section{Notation and terms}

Unless specified otherwise, an italic letter (e.g.T, $h_{k, n}$ ) represents a scalar and its bold face lower-case letter represents its corresponding vector (e.g. $\mathbf{h}_{k}=$ $\left.\left[h_{k, 1} h_{k, 2}, h_{k, N_{p}}\right]\right)$. A bold face upper-case letter (e.g. A) represents a matrix. The subscriber $\boldsymbol{k}$ denotes the time index of an OFDM symbol, $\boldsymbol{n}$ denotes the index of subcarriers in the frequency domain, $\boldsymbol{l}$ denotes the lth path of the radio channel. $|x|(|A|)$ is the elementwise magnitude of a vector x (matrix $\mathbf{A}$ ). $\mathbf{I}_{N}$ is an $N \times N$ identity matrix. $\mathbf{A}_{i, j}$ denotes the entry at the $i$ th row and the $\boldsymbol{k}$ th column of $\mathbf{A}$.

$L$ denotes the total number of possible paths in a radio channel, referred to as channel length, $N$ denotes the total number of subcarriers, $N_{p}$ the number of pilot subcarriers, $g_{k, l}$ the channel impulse response (CIR) of $\boldsymbol{l}$ th path at $\boldsymbol{k}$ th symbol, referred to as tap, $\mathbf{g}_{k}$ the CIR vector 
at $k$ th symbol time, $\mathbf{g}_{k}=\left[g_{k, 1} \cdots h_{k, L}\right], h_{k, n}$ the channel frequency response (CFR) at $\boldsymbol{k}$ th symbol time and $\boldsymbol{n}$ th subcarrier, $\overline{\mathbf{h}}_{k}$ the CFR vector at all subcarriers at $\boldsymbol{k}$ th symbol time, $\overline{\mathbf{h}}_{k}=\left[h_{k, 1} \cdots h_{k, N}\right], \mathbf{h}_{k}$ the CFR vector at $N_{p}$ pilot subcarriers at $\boldsymbol{k}$ th symbol time, $\mathbf{h}_{k}=\left[h_{k, 1} \cdots h_{k, N_{p}}\right]$, $\mathbf{a}_{k}$ the time-correlation coefficients of CFR at $k$ th symbol time, $\mathbf{x}_{k}$ the vector of transmitted OFDM symbols at pilot subcarriers at $\boldsymbol{k}$ th symbol time and $\mathbf{y}_{k}$ the corresponding received OFDM symbol vector of $\mathbf{x}_{k}$.

\section{System model}

Figure 1 describes the LTE downlink baseband system used in this article. Here, we only consider baseband processing and omit all analogue components, higher layer protocols and application processing. The baseband processor receives the digitized signal as complex samples from the analogue-to-digital convertors and posts the decoded data stream to the higher layer protocol and the application processor.

\section{Pilot symbols in LTE downlink}

As depicted in Figure 1, a radio frame of the LTE downlink has duration of $10 \mathrm{~ms}$ and consists of ten subframes each of $1 \mathrm{~ms}$. Each subframes has two 0.5 -ms time slots with each slot consisting of $N_{s y m b}^{D L}$ OFDM symbols (the values of $N_{\text {symb }}^{D L}$ for various configurations are given in Table 1). The transmitted downlink signal is represented as a time-frequency resource grid. Each small box within the grid represents a single subcarrier for one symbol
Table 1 Physical RBs parameters

\begin{tabular}{llll}
\hline Configuration & & $\mathbf{N}_{\mathbf{s c}}^{\mathbf{R B}}$ & $\mathbf{N}_{\text {symb }}^{\text {DL }}$ \\
\hline Normal cyclic prefix & $\Delta f=15 \mathrm{kHz}$ & 12 & 7 \\
Extended cyclic & $\Delta f=15 \mathrm{kHz}$ & & 6 \\
prefix & $\Delta f=7.5 \mathrm{kHz}$ & 24 & 3 \\
\hline
\end{tabular}

period and is referred to as a resource element. Note that in MIMO applications, there is a resource element mapping graph for each transmitting antenna. A resource block (RB) is defined as consisting of $N_{s c}^{R B}$ consecutive subcarriers for one slot ( $N_{\text {symb }}^{D L}$ OFDM symbols). An RB is the smallest unit of bandwidth-time resource allocation assigned by the base station scheduler, and the specification for the parameters of one RB is shown in Table 1.

In order to successfully receive a data transmission, the receiver must estimate the CIR to mitigate the multi-path interference. In packet-oriented networks (like IEEE 802.11), a physical preamble is used to facilitate this purpose. In contrast to 802.11, LTE makes use of PSAM, where known reference symbols, referred to as pilot symbols, are inserted into the stream of data symbols, as shown in Figure 1. Generally, there are three kinds of time-frequency allocation pattern of pilot symbols, namely, entirely known OFDM symbols, pilot subcarriers and scattered pilots. 3GPP LTE adopts a scattered pattern involving the sparse insertion of known pilot symbols in a data symbol stream. For example, in the scenario of a single transmitting and a single

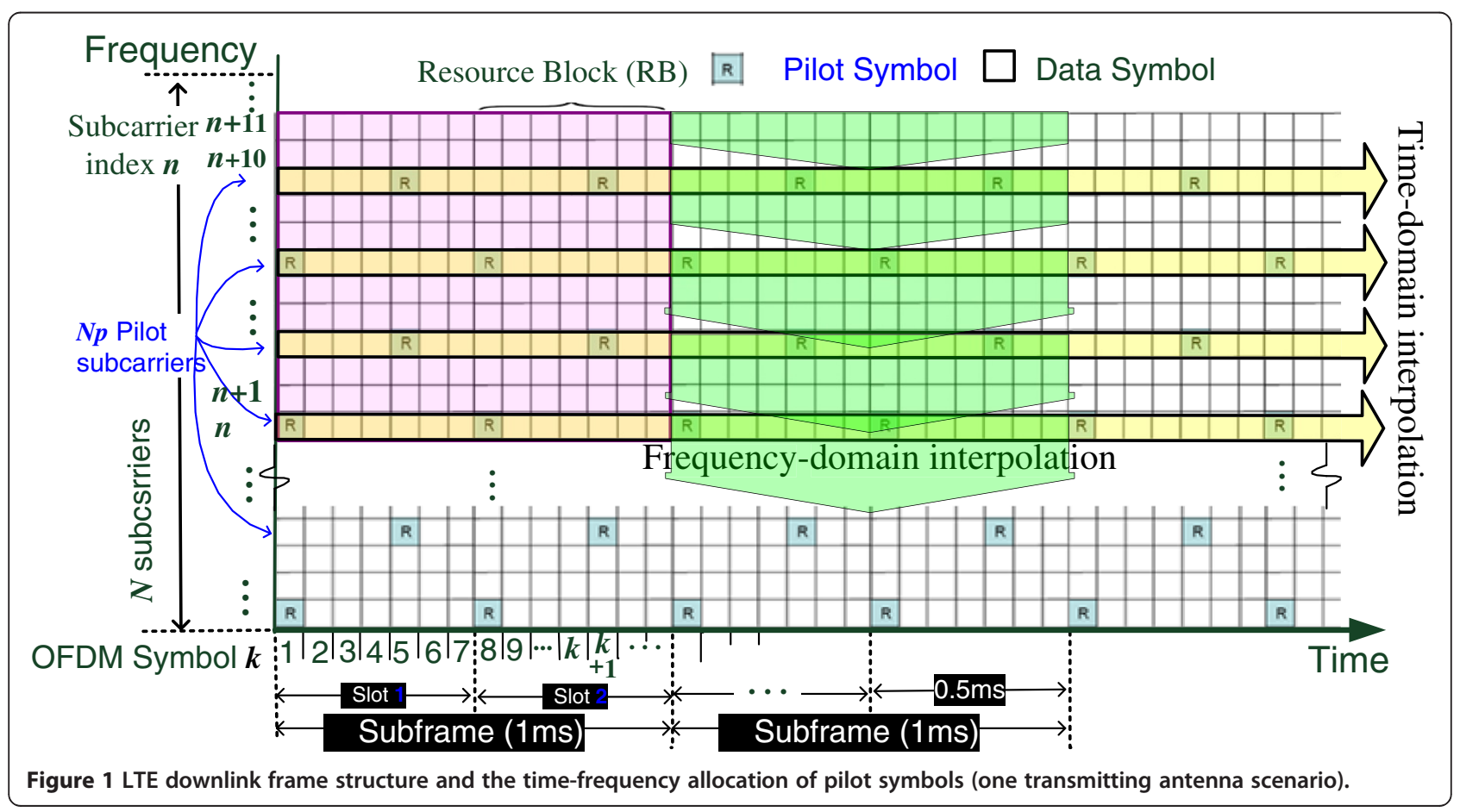


receiving antenna, pilot symbols are transmitted at the first and the fifth OFDM symbols of each slot at the pilot subcarriers. In the frequency domain, reference signals are spread over every six subcarriers.

The effect of the channel response on the known pilot symbols can be computed directly by calculating the attenuation of each pilot symbol [5]. For the remaining unknown data symbols, interpolation has to be used to estimate the channel response among adjacent pilot symbols. A simple way of performing this interpolation is the linear approximation in both time and frequency. The concept of PSAM in OFDM systems allows the use of both the time and frequency correlation properties of the channel to improve the channel estimation. Therefore, an efficient channel estimation procedure may apply a complicated 2D time-frequency interpolation or a combination of two simple 1D interpolations [6] to provide an accurate estimation of the channel states for each OFDM symbol.

\section{Channel model}

In this article, we consider an LTE downlink system with $N$ subcarriers over a Rayleigh-fading channel. For the purpose of analysis, the following notation and assumptions are taken in this article.

(1)The system bandwidth is $B=1 / T$, where $T$ is the duration of one time-chip. The duration of one OFDM symbol is $T_{s}=N T+T_{C P}$, where $T_{\mathrm{CP}}$ is the duration of cyclic prefix (CP) for every OFDM symbol.

(2) The number of possible path is $L$ and the maximum delay due to multi-path is $(L-1) T$.

(3) The length of $\mathrm{CP}$ is carefully designed to eliminate inter-symbol interference between consecutive OFDM symbols. That is $T_{\mathrm{CP}}$ is longer than the than the channel's maximum delay, $(L-1) T<T_{C P}$.

(4) The Rayleigh-fading channel varies in consecutive OFDM symbols, but is assumed constant within one OFDM symbol.

The time-varying multi-path channel can be represented in the continuous time-domain function by a collection of paths

$$
g(t, \tau)=\sum_{l=0}^{L-1} \alpha_{1}(t) \delta\left(\tau-\tau_{l}\right)
$$

where the $l$ th path is represented by a tap with complex amplitude $\alpha_{l}(t)$ at time instant $t$ and a delay $\tau_{l}$. The impulse response of the physical channel consists of independent Rayleigh-fading impulses, uniformly distributed over the length of the CP.
In the OFDM implementation of the 3GPP LTE, the transmitted and received signals are sampled for D/A and A/D conversion with an interval of chip duration $T$, the CIR (1) in the continuous time-domain is converted into an equivalent discrete channel model with sampling interval $T$. We define $g_{k, l}=\alpha_{l}(k T)$ as representing the complex magnitude of the $l$ th path with delay $l T$ during the $\boldsymbol{k}$ th OFDM symbol. The equivalent discrete model of the radio channel (1) is therefore

$$
\mathbf{g}_{k}=\sum_{l=0}^{L-1} g_{k, l} \delta(\tau-l T)
$$

Hence, the discrete CIR model can be represented by a length- $\boldsymbol{L}$ CIR vector $\mathbf{g}_{k}$

$$
\mathbf{g}_{k}=\left[g_{k, 0} g_{k, 1} \cdots g_{k, L-1}\right]^{T}
$$

Strictly speaking, $\mathbf{g}_{k}$ is only an approximation of $g(t, \tau)$ at $k$ th OFDM symbol $(k T s<t<(k+1) T s)$. When the multipath taps do not fall in the discrete sampling grid (i.e., $\tau_{l} \neq l T$ ), the discrete-time CIR vector will be infinite in length. However, the pulse's energy decays quickly outside the neighbourhood of the original pulse location $[5,14]$, it is still feasible to capture the impulses with a length- $\boldsymbol{L}$ vector. In this study, we assume that the tails of the impulse response function are negligible beyond $L$ samples, which is also the assumption made in OFDM to justify that no ISI occurs.

In the frequency-domain, the frequency response of the CIR $\mathrm{g}_{k}$ at $\boldsymbol{k}$ th OFDM symbol is

$$
\overline{\mathrm{h}}_{k}=\left[h_{k}[1] h_{k}[2] \cdots h_{k}[N]\right]^{T}
$$

where $\left\{h_{k}[n]\right\}, \quad n \in\{1,2, \ldots N\}$, denoting the CFR of $\boldsymbol{n}$ th subcarrier at $\boldsymbol{k}$ th OFDM symbol time is converted from the time-domain CIR via the discrete Fourier transform (DFT)

$$
h_{k}[n]=\sum_{l=0}^{L-1} g_{m}[l] e^{-j 2 \pi \frac{n-1}{N} l}
$$

The relationship between the CIR in time-domain and CFR in frequency domain can be described in matrix notation

$$
\overline{\mathbf{h}}_{k}=\mathbf{F}_{L} g_{k}
$$

where $F_{L} \in \mathbb{C}^{N \times L}$ is the first $L$ columns of the $N \times N$ DFT matrices $\mathbf{F}$. And $\mathbf{F}$ is denoted by

$$
F_{j, k}=\frac{1}{\sqrt{N}} e^{-j 2 \pi(j-1)(k-1) / N}, \ldots(1 \leq j, k \leq N)
$$

It has been shown that time-varying path taps in a fading channel can be modelled by an AR process $[11,15]$, which is applicable to general fading channels, and in 
particular to mobile communication. Examples include the first-order AR model in $[1,11,16]$ and the secondorder AR model [15]. Although the first-order AR model is just an approximation to the actual statistics of the random radio propagation process, it is more realistic than those models assuming constant channel parameters (identity matrix) or using linear interpolation. Furthermore, the use of higher-order models will lead to higher computational costs, which may not be justified by the performance improvement. Compared to the higher-order model, a lower-order model may reduce the overall computational complexity significantly with only a relatively small performance sacrifice. Here, we are concerned with the basic derivation of the proposed Kalman interpolator filter in LTE downlink. As shown in our following derivation, higher-order models can also be incorporated into the proposed scheme with only minor modifications. For the purpose of analysis, we restrict ourselves to a firstorder AR model for the time-varying channel.

It is easy to verify that the channel coefficients $\overline{\boldsymbol{h}}_{k}$ of the time-varying CFR can be modelled by the following dynamic AR process $[1,11,12]$ :

$$
h_{k+1, n}=a_{n} h_{k, n}+v_{k, n},(n=1,2, \ldots \mathrm{N})
$$

where $\alpha_{n}$ represents the time correlation of the channel response between $\boldsymbol{k}$ th and $(\boldsymbol{k}+1)$ th OFDM symbols at the $n$th subcarrier. $v_{k, n}$ is a mutually independent zeromean Gaussian complex white noise representing the modelling error.

\section{LTE OFDM reception and channel estimation}

In order to estimate the CFR as defined in (4), $N_{p}$ pilot symbols are inserted sparsely among $\boldsymbol{N}$ subcarriers at $\boldsymbol{k}$ th OFDM symbol duration following the comb pattern shown in Figure 1. Let $\mathbf{x}_{k}=\left[x_{k, 1}, x_{k, 2}, \ldots, x_{k, N_{p}}\right]^{T} \in \mathbb{C}^{N_{p}}$ denote the transmitted pilot vector of $N_{p}$ known pilot symbols at the $k$ th OFDM symbol, $\mathbf{y}_{k}=$ $\left[y_{k, 1}, y_{k, 2}, \ldots, y_{k, N_{p}}\right]^{T} \in \mathbb{C}^{N_{p}}$ denotes the vector of the received pilot symbols. After CP removal, the received pilot symbols can be expressed as

$$
\mathbf{y}_{k}=\mathbf{X}_{k} \mathbf{h}_{k}+\mathbf{w}_{k}
$$

where $\mathbf{X}_{k}=\operatorname{diag}\left(x_{k}\right)$ is an $N_{p} \times N_{p}$ diagonal matrix with transmitted pilot symbols $\mathbf{x}_{k}$ as its diagonal elements,

$$
\mathbf{X}_{k}=\operatorname{diag}\left(x_{k}\right)=\left[\begin{array}{ccccc}
x_{k, 1} & 0 & \ldots & 0 \\
0 & x_{k, 2} & 0 & \ldots & 0 \\
0 & \vdots & 0 & \ldots & 0 \\
0 & 0 & \ldots & x_{k, N_{p}}
\end{array}\right]
$$

Here, $w_{k} \in \mathbb{C}^{N_{p}}$ is an additive white complex Gaussian noise with covariance matrix $\sigma_{w}^{2} I_{N_{p}}$ and $h_{k} \in \mathbb{C}^{N_{p}}$ is the CFR at pilot subcarriers at $\boldsymbol{k}$-th OFDM symbol.
The goal of channel estimation is to estimate the whole CFR for all data carriers from $\mathbf{h}_{k}$ at these $N_{p}$ pilot symbols with as high accuracy as possible. This is an optimization problem described as

$$
\arg \min _{\mathbf{h}} \sum_{k}\left\|\mathbf{x}_{k}-\mathbf{y}_{k} \cdot / \mathbf{h}_{k}\right\|_{2}
$$

where $\mathbf{y}_{k} / \mathbf{h}_{k}$ is an element-wise division with elements $\mathbf{y}_{k, n} / \mathbf{h}_{k, n}$.

It is worth noting that, as the pilot symbols in LTE downlink are inserted into the data symbols sparsely in a frequency-time scatter pattern, the channel response at data symbols are typically interpolated from the channel estimates at pilot symbols. As shown in literature, if the OFDM symbol is short compared with the coherence time of the channel, the time correlation between the channel attenuation of consecutive OFDM symbols is high. There is also a substantial frequency correlation between the channel attenuation of adjacent subcarriers. For a better channel estimation at data symbols, both of these time and frequency correlation properties of the fading channel can be exploited by the channel estimator.

Generally, as illustrated in Figure 1, the whole process of such a pilot-aided channel estimation includes three steps: (1) Estimation at pilot symbols, where, $\mathbf{h}_{\boldsymbol{k}}$, the channel responses at $N_{p}$ pilot subcarriers at $\boldsymbol{k}$ th OFDM symbol are calculated with the common LS estimator or LMMSE estimator; (2) Time-domain interpolation, where the channel responses $\mathbf{h}_{k+1}$ at $(\boldsymbol{k}+1)$ th OFDM symbol at pilot subcarriers are estimated from $\mathbf{h}_{k}$ by tracking the parameters of each path. (3) Frequency-domain interpolation, where the channel responses at all $N$ subcarriers are estimated by interpolating or smoothing these estimates $\left\{\mathbf{h}_{k}, \mathbf{h}_{k+1}, \ldots\right\}$ at pilots subcarriers. This article integrates the first two steps into one framework called the Kalman interpolator filter.

\section{EKF for channel estimation}

In this section, we are interested in deriving a minimum variance estimator/interpolator for the channel response $\left\{\mathbf{h}_{k}, \mathbf{h}_{k+1}, \ldots\right\}$ at pilot subcarriers from the observation of sparse pilot symbols. We present a combined estimation and interpolation scheme, where the time correlation among consecutive OFDM symbols is taken into account to estimate the CFR at the known pilot symbols and then to interpolate to estimate the CFR at the unknown data symbols at the pilot subcarriers. The proposed scheme is based on the idea of Kalman filtering to improve the accuracy of the estimation and interpolation. More specifically, recalling the LTE 
reception model $y_{k}=X_{k} h_{k}+w_{k}$ in (9), the task for the Kalman interpolator filter can be stated as:

Given the matrix $\mathrm{X}_{\mathrm{k}}$ of known transmitted pilot symbols and received signal $\mathrm{y}_{k}$ at $k$ th OFDM symbol, to obtain minimum variance estimates of the time-varying multipath CFR $\mathrm{h}_{k}$ and interpolate $\mathrm{h}_{k}$ to the followed six $\{k+1, k+2, \ldots, k+6\}$ data symbols at the pilot subcarriers until the next pilot symbol $(k+7$-th OFDM symbol) is received.

\section{Augmented state space model}

Considering a time-varying channel described in Equation (8), the CFR at pilot subcarriers can be described as a state space model

$$
\left\{\begin{array}{c}
h_{k+1}=A_{k} h_{k}+v_{k} \\
y_{k}=X_{k} h_{k}=w_{k}
\end{array}\right.
$$

where $\mathbf{h}_{k}$ is the state variable to be estimated, $A_{k} \in$ $\mathbb{C}^{N_{p} \times N_{p}}$ is the unknown state transition matrix consisting of the time correlation coefficients $\alpha_{n}$ of channel response. Both $\mathbf{v}_{k}$ and $\mathbf{w}_{k}$ are mutually independent, zero-mean, Gaussian complex white noises, with covariance $Q_{v} \triangleq \sigma_{\mathrm{w}}^{2} I_{\mathrm{N}_{\mathrm{p}}}$ and $Q_{w} \triangleq \sigma_{\mathrm{w}}^{2} I_{\mathrm{N}_{\mathrm{p}}}$, respectively. It is assumed that $\mathbf{v}_{k}$ and $\mathbf{w}_{k}$ are independent of the state variable $\mathbf{h}_{k}$. Note that, in this state space model of the CFR, the state transition matrix $\mathbf{A}_{k}$ is unknown and to be estimated together with the state variable $\mathbf{h}_{k}$. Therefore, it is a problem of joint state and parameter estimation. The purpose is to estimate both the channel response $\mathbf{h}_{k}$ and channel's time-correlation matrix $\mathbf{A}_{k}$ from the received pilot symbols $\mathbf{y}_{k}$.

Considering that $\mathbf{A}_{k}$ is a spares matrix in most cases, without loss of generality, we assume $\mathbf{A}_{k}$ has $N_{A}$ $\left(N_{A}<N_{p} \times N_{p}\right)$ unknown entries to be estimated and let a vector $\mathbf{a}_{k}$ denote all the $N_{A}$ unknown entries as follows:

$$
\mathbf{a}_{k} \triangleq \operatorname{Vec}\left(A_{k}\right)
$$

$\mathbf{a}_{k} \in \mathbb{C}^{N_{A}}$ is an $N_{A} \times 1$ column vector formed by stacking all unknown entries of the matrix $\mathbf{A}_{k}$ in a row-wise order. The time-correlation parameters are now represented by $\mathbf{a}_{k}$ which is the vector to be estimated. For the purpose of clarification, $\mathbf{A}_{k}$ is represented by $\mathbf{A}\left(\mathbf{a}_{k}\right)$ explicitly in the following. Assuming a random walk model for the parameter $\mathbf{a}_{k}$, then Equation (12) becomes

$$
\left\{\begin{array}{l}
a_{k+1}=a_{k}+\epsilon_{k} \\
h_{k+1}=A\left(a_{k}\right) h_{k}+v_{k} \\
y_{k=1}=X_{k} h_{k}+w_{k}
\end{array}\right.
$$

where $\epsilon_{k}$ denotes the process noise of $\mathbf{a}_{k}$ and is an independent, zero-mean Gaussian noise with covariance
$Q_{\epsilon} \triangleq \sigma_{\epsilon}^{2} I_{N_{A}}$. In order to jointly estimate the state and parameters, a new augmented state $\mathbf{z}_{k}$ is defined as

$$
\mathbf{z}_{k}=\left[\begin{array}{ll}
\mathbf{a}_{k}^{T} & \mathbf{h}_{k}^{T}
\end{array}\right]^{T}
$$

and the channel state space model Equation (14) turns into an augmented system

$$
\left\{\begin{array}{l}
z_{k+1}=f\left(z_{k}\right)+u_{k} \\
y_{k}=\left[0_{1 \times N_{A}} \quad X_{k}\right] z_{k}+w_{k}
\end{array}\right.
$$

where $u_{k}=\left[\epsilon_{k}^{T} v_{k}^{T}\right]^{T}$ with covariance matrix $Q_{u}=$ $\left[\begin{array}{cc}Q_{\sigma} & 0 \\ 0 & Q_{\nu}\end{array}\right]$ and $f\left(\mathbf{z}_{\mathbf{k}}\right)$ is the nonlinear state transition function

$$
f\left(\mathbf{z}_{k}\right)=\left[\begin{array}{c}
\mathbf{a}_{k} \\
\mathbf{A}\left(\mathbf{a}_{k}\right) \mathbf{h}_{k}
\end{array}\right]
$$

\section{EKF}

Since the state transition function $f\left(\mathbf{z}_{n}\right)$ in the augmented state model (16) is a nonlinear function and an EKF has to be used to estimate the augmented states. The development of the EKF basically consists of two procedures: linearizing the augmented model (16) and applying the standard KF to the linearized model.

The linearization procedure is included in the Appendix where the derivation of the EKF algorithm for a general matrix $\mathbf{A}\left(\mathbf{a}_{k}\right)$ is demonstrated. The basic concept is to form the Taylor approximation of the nonlinear transition function. The resulting linear state space model approximating the AR model (12) is

$$
\left\{\begin{array}{l}
\mathbf{z}_{k+1}=\mathrm{F}_{k} \mathbf{z}_{k}+\mathbf{u}_{k} \\
\mathbf{y}_{k}=\left[\begin{array}{ll}
\mathbf{0} & \mathbf{X}_{k}
\end{array}\right] \mathbf{z}_{k}+\mathbf{w}_{k}
\end{array}\right.
$$

Applying the standard KF to the model (18) is straightforward. The resulting EKF algorithm for the joint estimation of CFR $\mathbf{h}_{k}$ and CFR's time correlation coefficients $\mathbf{a}_{k}$ works in an iterative prediction-correction cycle. The prediction projects forward (in time) the current estimate $\hat{z}_{k}$ and error covariance $\mathbf{P}_{k}$ at $\boldsymbol{k}$ th OFDM symbol to obtain the a priori estimates $\hat{z}_{k+1 \mid k}$ and $P_{k+1 \mid k}$ for the next $(\boldsymbol{k}+1)$ th OFDM symbol. The correction adjusts the projected estimates $\hat{z}_{k+1 \mid k}$ and $P_{k+1 \mid k}$ to obtain an improved a posteriori estimate by using an actual measurement of received symbol $\mathbf{y}_{\boldsymbol{k}+1}$ at $(\boldsymbol{k}+1)$ th OFDM symbol. Here, the subscript $k+1 \mid k$ corresponds to one-step a priori prediction, $k \mid k$ corresponds to a posteriori correction and $k \mid k$ is denoted by $k$ for the purpose of short notation. More specifically, the filtering algorithm is presented as follows: 
1. Prediction (before receiving a OFDM symbol):

$$
\begin{aligned}
\hat{z}_{k \mid k-1} & =f\left(z_{k-1}\right)=\left[\begin{array}{c}
\hat{a}_{k-1} \\
\hat{A}_{k-1} \hat{h}_{k-1}
\end{array}\right] \\
P_{k \mid k-1} & =F_{k-1} P_{k-1} F_{k-1}^{H}+Q_{u} \\
& =\left[\begin{array}{cc}
P_{a, k \mid k-1} & P_{a h, k \mid k-1} \\
P_{a h, k \mid k-1}^{H} & P_{h, k \mid k-1}^{H}
\end{array}\right]
\end{aligned}
$$

where

$$
\begin{aligned}
\mathbf{P}_{a, k \mid k-1}= & \mathbf{P}_{a, k-1}+\mathbf{Q}_{\in, k} \\
\mathbf{P}_{a h, k \mid k-1}= & \mathbf{P}_{a, k-1} \hat{\mathbf{H}}_{k-1}^{H}+\mathbf{P}_{a h, k-1} \hat{\mathbf{A}}_{k-1}^{H} \\
\mathbf{P}_{h, k \mid k-1}= & \hat{\mathbf{H}}_{k-1} \mathbf{P}_{a, k-1} \hat{\mathbf{H}}_{k-1}^{H}+\hat{\mathbf{A}}_{k-1} \mathbf{P}_{h, k-1} \hat{\mathbf{A}}_{k-1} \\
& +\hat{\mathbf{H}}_{k-1} \mathbf{P}_{a h, k-1} \hat{\mathbf{A}}^{H}+\hat{\mathbf{A}}_{k-1} \mathbf{P}_{a h, k-1} \hat{\mathbf{H}}_{k-1}^{H}+\mathbf{Q}_{v, k}
\end{aligned}
$$

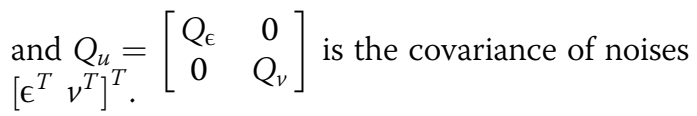

2. Correction (once the reception of the OFDM symbol has completed):

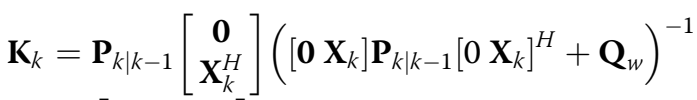

$$
\begin{aligned}
& =\left[\begin{array}{c}
\mathbf{P}_{a h, k \mid k-1} \\
\mathbf{P}_{h, k \mid k-1}
\end{array}\right] \mathbf{X}_{k}^{H}\left(\mathbf{X}_{k} \mathbf{P}_{h, k \mid k-1} \mathbf{X}_{k}^{H}+\mathbf{Q}_{w}\right)^{-1} \\
& \hat{\mathbf{z}}_{k}=\hat{\mathbf{z}}_{k \mid k-1}+\mathbf{K}_{k}\left(\mathbf{y}_{k}-\left[\begin{array}{l}
\mathbf{0} \\
\mathbf{X}_{k}
\end{array}\right] \hat{\mathbf{z}}_{k \mid k-1}\right) \\
& =\hat{\mathbf{z}}_{k \mid k-1}+\mathbf{K}_{k}\left(\mathbf{y}_{k}-\mathbf{X}_{k} \hat{\mathbf{h}}_{\boldsymbol{k} \mid k-1}\right) \\
& P_{k}=P_{k \mid k-1}-K_{k}\left[\begin{array}{ll}
0 & X_{k}
\end{array}\right] P_{k \mid k-1}
\end{aligned}
$$

Here, $\mathbf{K}_{k}$ is the Kalman gain of the EKF. The EKF makes use of a first-order Taylor approximation of the state transition and thus does not approach the true minimum variance estimate when the linearization error is non-negligible. Nevertheless, the resulting EKF is a practical approximation to the minimum variance estimator when the state equation is nonlinear, and will be shown to provide a good performance in time-varying channel estimation. Furthermore, the EKF has successfully been applied to the problem of joint channel state and parameter estimation in $[11,16]$, and thus it seems reasonable to apply EKF to the time-varying channel estimation.

\section{Remark}

In terms of computation complexity, it can be seen that prediction of state error covariance $\mathbf{P}_{k+1 \mid k}$ and the update of $\mathbf{K}_{k}$ consumes the major amount of computation. Fortunately, in general, cross-path coupling is confined within a small neighbourhood, and thus the off-diagonal elements of $\mathbf{A}_{k}$ representing the coupling between multiple paths are small and may be neglected. As shown in the AR model (8) of time-varying channel, the channel's time-correlation matrix $\mathbf{A}_{k}$ can be modelled as a diagonal matrix. If both $\mathbf{X}_{k}$ and $\mathbf{A}_{k}$ are diagonal matrices, the number of complex multiplications and additions is be reduced to a great extent. More specifically, the number of multiplication and division operations in Equations (19)-(24) is $25 N_{p}$.

\section{EKF for channel interpolation}

In this section, the proposed EKF is further extended to interpolate the CFR estimate to unknown data symbols and the whole estimation and interpolation process of the proposed EKF is summarized.

Figure 2 illustrates the block diagram of the baseband channel model and the proposed Kalman interpolation filter for LTE downlink channel equalizer. The EKF works in an iterative prediction-correction manner and, in the application of LTE downlink channel estimation, each iteration corresponds to the duration of an OFDM symbol. However, due to the fact that the known pilot symbols are inserted sparsely into the unknown data symbols, the coefficient matrix $\mathbf{X}_{k}$ is not always available at each iteration. Like most adaptive algorithms, two working modes, namely, training mode and decisiondirected interpolation mode, are adopted in the proposed Kalman interpolation filter to address this issue.

The estimator is trained during these periods when a pilot symbol is received. Then it switches to an interpolation mode, in which a decision-directed method is applied to estimate the channel response until the next pilot symbol is received. During the training period, the transmitted symbols $\mathbf{X}_{k}$ are known to the estimator, while in the data symbols periods, the transmitted data symbols are estimated as $\hat{\mathbf{X}}_{k}$ by the decoder and the EKF is fed by the $\hat{\mathbf{X}}_{k}$ to replace the unknown transmitted symbols $\mathbf{X}_{k}$. Indeed, the channel estimator is fed with one pilot symbol and six estimates of the data symbols in one LTE slot. The proposed Kalman interpolator filter method yields an adaptive algorithm and can be implemented recursively.

At each iteration, the equalizer and the decoder compute an estimate $\hat{\mathbf{X}}_{k}$ of the transmitted data symbols on the basis of the previous, a priori channel estimate $\hat{h}_{k \mid k-1}$. In the iteration of the OFDM data symbol, $\hat{\mathbf{X}}_{k}$ is also fed to the EKF to calculate a posteriori channel estimate $\hat{h}_{k \mid k}$ and a priori channel estimate $\hat{h}_{k+1 \mid k}$. By exchanging their estimates, both EKF and equalizer are able to improve their performance iteratively. This is particularly useful at these iterations of unknown data symbols.

\section{Initialization by LS estimation}

Although a KF is able to convergence under any reasonable initial value of the state variable $\mathbf{z}_{k}$, a good initial 


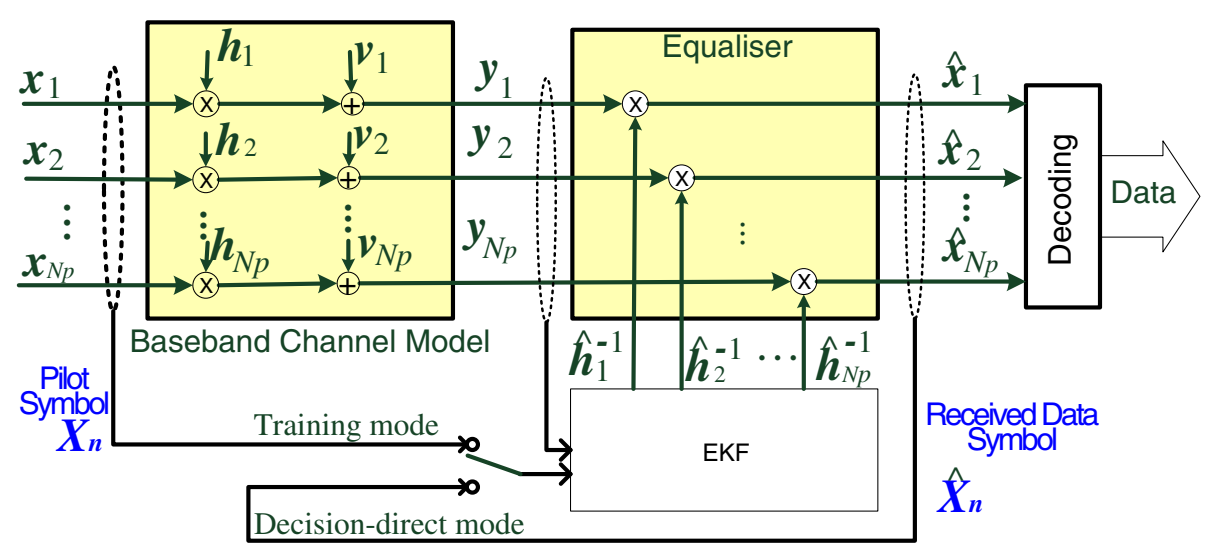

Figure 2 Channel estimation and interpolation at pilot subcarriers.

condition will reduce the duration of convergence. Generally, if the initial value of the state variable is set to the neighbourhood of the true value, a faster convergence can be obtained. Since the state variable $\mathbf{z}_{k}$ consists of two independent components, $\mathbf{a}_{k}$ and $\mathbf{h}_{k}$, their initial values are chosen separately.

For initializing the channel's time-correlation coefficients $\mathbf{a}_{k}$, we use an identity matrix $\left(A\left(a_{0}\right)=I_{N_{A}}\right)$ assuming the channel response at next OFDM symbol is the same as the current OFDM symbol. Although an identity matrix represents a time-invariant channel, an identity matrix would be the best choice of the channel's initial condition, given we have no a priori knowledge about the channel.

For CFR $\mathbf{h}_{k}$, we shall use the conventional version of an LS estimation to get the initial value. When the first group of pilot symbols is received, the LS method is performed as follows:

$$
\begin{aligned}
\hat{h}_{0, L S} & =\left(X_{0}^{H} X_{0}\right)^{-1} X_{0}^{H} y_{0} \\
& =\left[\frac{y_{0,1}}{x_{0,1}}, \frac{y_{0,2}}{x_{0,2}}, \ldots, \frac{y_{0}, N_{p}}{x_{0}, N_{p}}\right]
\end{aligned}
$$

where $\hat{h}_{0, L S} \in \mathbb{C}^{N_{p}}$ is the initial CFR estimate. A more complicated LMMSE estimator using the channel's frequency correlation may be applied to obtain a more accurate initial estimate of the CFR. It should be pointed out that the EKF is initialized until the first group of pilot symbol is received.

\section{Trained estimation}

After the state variable is initialized, the EKF works iteratively either in the training mode or in the interpolation mode. During the pilot symbols, the EKF switches to the training mode, where the known pilot symbol forms the matrix $\mathbf{X}_{k}$. As the observation $\mathbf{y}_{k}$ is obtained by the DFT at the end of an OFDM symbol duration, the a posteriori CFR $\hat{h}_{k \mid k}$ is first estimated from $\mathbf{y}_{k}$ by using update equations (22)-(24). Then the a priori estimate $\hat{h}_{k+1 \mid k}$ is calculated by Equations (19)(21) for next OFDM symbol.

\section{Decision-directed interpolation}

During periods where the pilot symbol is not available, the EKF switches to decision-directed interpolation mode to continue adaptation. For these data symbols, as the transmitted symbol $\mathbf{X}_{k}$ is unknown, $\mathbf{X}_{k}$ is replaced by the decoder's decision of $\hat{X}_{k}$ that is supposed to be nearest to $\mathbf{X}_{k}$. In the decision-directed mode, the prediction and correction processes are the same as the training mode, except $\mathbf{X}_{k}$ is replaced by $\hat{X}_{k}$.

It is worth noting that, as $\mathbf{y}_{k}$ is only available at the end of the current symbol duration, the correction process has to be carried out at the end of the symbol duration. Thus, the equalizer uses the a priori channel estimate $\hat{h}_{k \mid k+1}$ to refine the currently received OFDM symbol, rather than uses the a posteriori CFR $\hat{h}_{k \mid k}$.

\section{Selection of the covariance matrices}

In most applications of Kalman filtering, it is difficult to measure the variance of noises. In practice, the covariance matrices are a priori approximated by applying the best available knowledge and tuned empirically in the application. As shown in the state space model (12), the channel measurement $\mathbf{y}_{k}$ is subject to the noise $\mathbf{w}_{k}$, the additive white complex Gaussian noise in the wireless channel. Since the transmission power and signal-to-noise ratio (SNR) are usually available in a communication system, the elements of the variance matrix $Q_{w}$ can be calculated by $\sigma_{w}^{2}=\frac{P_{t x}}{10^{S N R / 10}}$, where $P_{t x}$ is the transmission power measured in Watts and SNR is in $\mathrm{dB}$. Presuming a small process variance and linearization errors in (18), the values of $\sigma_{v}^{2}$ and $\sigma_{\epsilon}^{2}$ in $Q_{v}$ are empirically selected from $\{0.1,0.01,0.001\}$ according 
to the SNRs. At low SNRs, the channel estimate is less accurate due to large observation noise and thus a larger value is used for $Q_{u}$. At higher SNRs, a better channel estimation is expected and a smaller value is used for $Q_{u}$.

\section{Summary}

We now summarize the proposed method for channel estimation in LTE downlink:

Step 1. Initialize $\left[\begin{array}{ll}a_{0}^{T} & h_{0}^{T}\end{array}\right]^{T}$ when the first pilot symbol is received, make the first a priori prediction $\left[\begin{array}{ll}a_{1 \mid 0}^{T} & h_{1 \mid 0}^{T}\end{array}\right]^{T}$ for next OFDM symbol and set $k=1$; When a new OFDM symbol ( $k$ th symbol) has been received, repeat the following steps $2-6$.

Step 2. Calculate $y_{k}$ by using DFT

Step 3 . Estimate $\hat{x}_{k}$ by equalizing $\mathrm{y}_{k}$ with previous a posteriori $h_{k \mid k-1}$;

Step 4. If $\mathrm{y}_{k}$ is pilot symbol, set $\mathrm{X}_{k}$ by the known pilot symbol $\mathrm{x}_{k}$,else set $\mathrm{X}_{k}$ by the estimated data symbol $\hat{x}_{k}$, Step 5. Correct a posteriori state estimation $\left[a_{k \mid k}^{T} h_{k \mid k}^{T}\right]^{T}$ from $\mathrm{y}_{k \text { by }}(22)-(24)$.

Step 6. Time-interpolation: Predict a priori state estimate $\left[a_{k-1 \mid k}^{T} h_{k+1 \mid k}^{T}\right]^{T}$ by (19)-(21) for next symbol. Step 7. Frequency-interpolation: The CFR at data subcarriers for next symbol is interpolated using a DFT-based interpolation [8].

Step $8 . k=k+1$, wait for next symbol and goes back to step 2.

It can be seen that, the proposed KF-based channel estimation scheme is a combination of the estimator (for pilot symbols) and the interpolator (for data symbols). When the pilot symbol is available at $\boldsymbol{k}$ th iteration, a direct observation of the channel state is obtained and the EKF works at the training mode giving the optimal estimate of CFR in the sense of minimum variance. In the followed six $\{k+1, k+2, \ldots, k+6\}$ data symbols, the EKF interpolates the CFR in decision-directed model until the next pilot symbol ( $k+7$-th OFDM symbol) is received.

\section{Simulation results and performance analysis}

In this section, simulation is performed to validate the performance of the proposed Kalman interpolation filter for LTE downlink systems. A simplified rural area model defined by 3GPP [17] is adopted to configure the Rayleigh channel with additive white Gaussian noise and the parameters are listed in Table 2. The LTE downlink simulation parameters are listed in Table 3. The total number of subcarriers is 512 with 300 of them used for data/pilot transmission and a quadrature phase-shift keying (QPSK) modulation employed. For simplicity, the raw bits randomly generated are not coded with turbo coding schemes. In the single input single output
Table 2 The simplified rural area channel model

\begin{tabular}{lll}
\hline Tap number & delay $(\boldsymbol{\mu s})$ & $\begin{array}{l}\text { Average path } \\
\text { gains }(\mathbf{d B})\end{array}$ \\
\hline 1 & 0 & -2.748 \\
2 & 0.1302 & -4.413 \\
3 & 0.2604 & -11.052 \\
4 & 0.3906 & -18.500 \\
5 & 0.5208 & -18.276 \\
\hline
\end{tabular}

scenario, 100 of the 300 subcarriers are used for carrying pilot symbols during the pilot OFDM symbol time period. Three speeds of user equipment are simulated, namely, 50200 and $300 \mathrm{~km} / \mathrm{h}$. For each speed, the simulation is repeated 10 times (10 runs) in order to obtain reliable statistics and each simulation run simulates the transmitting/receiving four LTE downlink subframes containing 56 OFDM symbols.

Figure 3 illustrates a plot of the channel surface for the urban channel model at a moving speed of $200 \mathrm{~km} / \mathrm{h}$, where the Doppler frequency is around $480 \mathrm{~Hz}$. This plot shows the time-varying and frequency-selective nature of the channel gain and provides an image of the true values of the CFR. Studying the channel surface indicates that fluctuations in the frequency are clearly visible, but relatively smoothly varying in time, implying an AR process would be able to represent the channel's time correlation. The channel surface also suggests that a linear interpolation may not be good for such a nonlinear CFR.

The simulations are carried out at different noise levels with the SNR varying from 0 to $40 \mathrm{~dB}$ at a step size of $5 \mathrm{~dB}$. Figure 4 shows an example of the CFR estimation errors at the 100 pilot subcarriers in one simulation run (with 4 subframes containing 56 OFDM symbols) at $\mathrm{SNR}=20 \mathrm{~dB}$ and moving speed $20 \mathrm{~km} / \mathrm{h}$. Figure 4a depicts the CFR surface estimation error given by the proposed EKF scheme and Figure $4 \mathrm{~b}$ depicts the

Table 3 LTE downlink simulation parameters

\begin{tabular}{ll}
\hline Parameters & Values \\
\hline Bandwidth & $5 \mathrm{MHz}$ \\
Total number of RBs & 25 \\
Number of total subcarriers & 300 \\
Number of pilot subcarriers in Pilot OFDM symbols & 100 \\
Subcarrier spacing & $15 \mathrm{kHz}$ \\
CP length & $4.69 \mathrm{\mu s}$ \\
Slot duration & $0.5 \mathrm{~ms}$ \\
Sample Rate & $7.68 \mathrm{MHz}$ \\
FFT size & 512 \\
Modulation & QPSK \\
Velocity & $\{50,200\} \mathrm{km} / \mathrm{h}$ \\
\hline
\end{tabular}




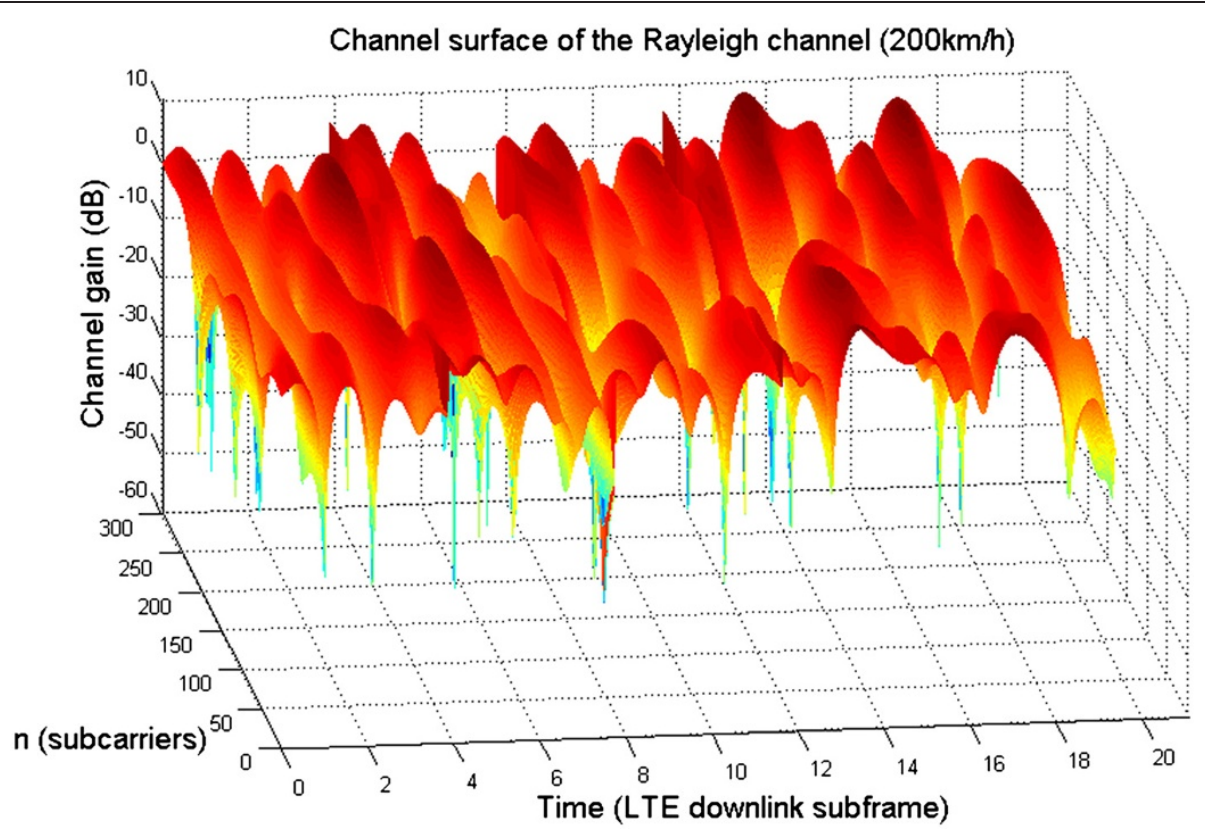

Figure 3 Channel surface of an LTE radio channel at a moving speed of $200 \mathrm{~km} / \mathrm{h}$.

estimation errors of the LS scheme, where the improvement of CFR estimation in the proposed scheme can be seen clearly and the mean square error (MSE) of the EKF is 0.066 and that of the LS is 0.09. The smaller CFR estimation error demonstrates the proposed EKF's ability to filter the noises in observation and to track the timevarying channel parameters. Particularly, towards the edge of the LTE downlink subframe (i.e. at the 14th, 28th, 42th, 56th OFDM symbols), a larger estimation error occurs in the LS estimation which can be seen clearly in Figure $4 \mathrm{~b}$. This is caused by the extrapolations errors in the LS scheme as no pilot symbols are inserted at the edge of each subframe. However, it is worth noting that the proposed EKF's estimation errors may have peaks at some data symbols, due to the fact that the incorrect data symbol decision is fed back to the EKF in the decision-directed mode. If a large deviation occurs and thus makes the received OFDM data symbol far from

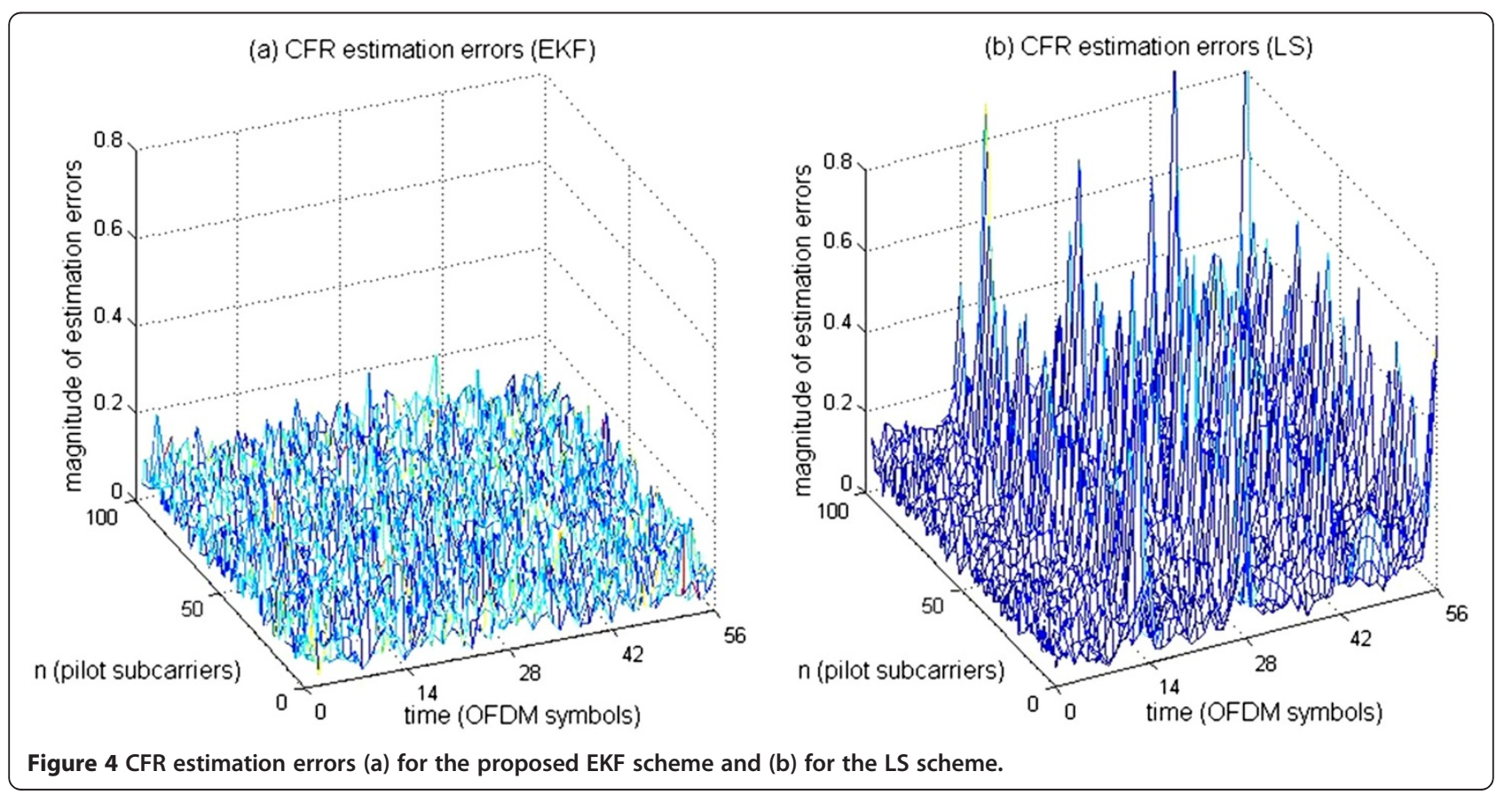




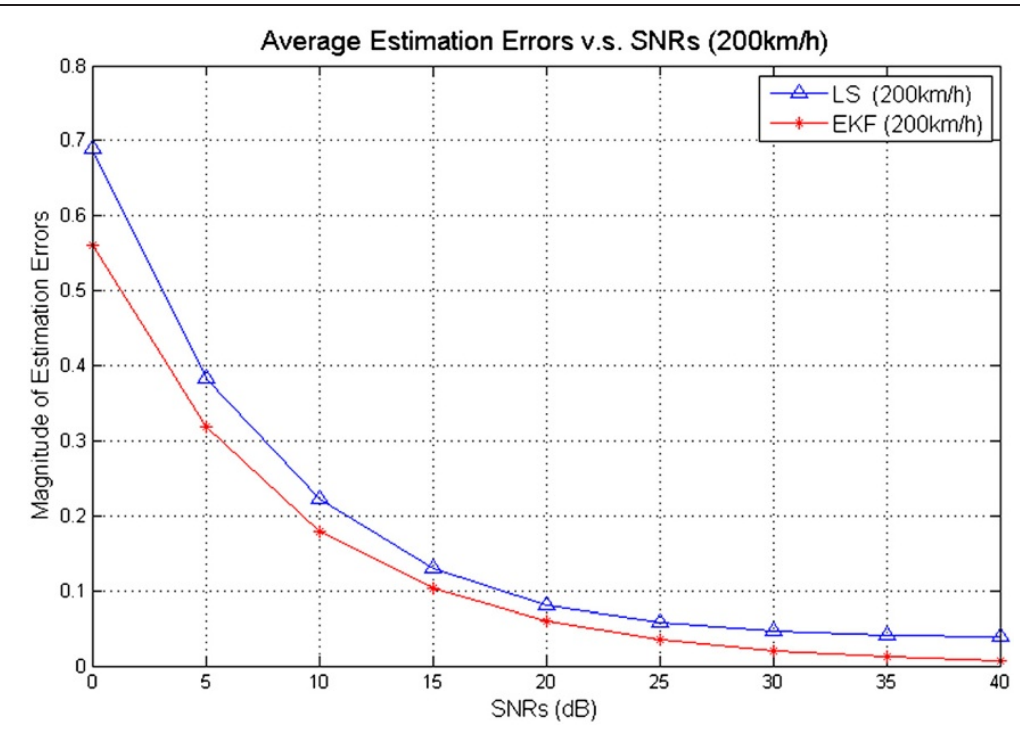

Figure 5 Average mean square estimation errors at various SNRs ( $200 \mathrm{~km} / \mathrm{h})$. Blue solid line with triangle: the average MSE of channel estimates by LS method. Red solid line with star: the average MSE of channel estimates by the proposed EKF method.

its original QAM constellation position but nearer to another constellation position, the quantization procedure will result in a wrong decision of the data symbol. When the incorrect data symbol decision is fed back to the EKF, it works as an incorrect 'observation' resulting in the EKF giving an abrupt change in state estimation. As a result, a sudden jump appears in the CFR estimates and may result in error propagation, making more errors in the following data symbol decision. If these decision errors are infrequent enough, the effects of these errors decay away and the decision-directed equalizer's performance remains similar to that of the training mode.

Figure 5 shows the average CFR estimation MSEs of the LS and EKF schemes at different SNRs. It can be seen that the EKF achieves a smaller estimation errors and gives a better CFR estimation.

The BER performances are plotted in Figures 6, 7 and 8 . Figure 6 is for low-speed environment $(50 \mathrm{~km} / \mathrm{h})$, Figures 7 and 8 are for high-speed environment (200 and $300 \mathrm{~km} / \mathrm{h}$ ), respectively. In the BER comparisons,

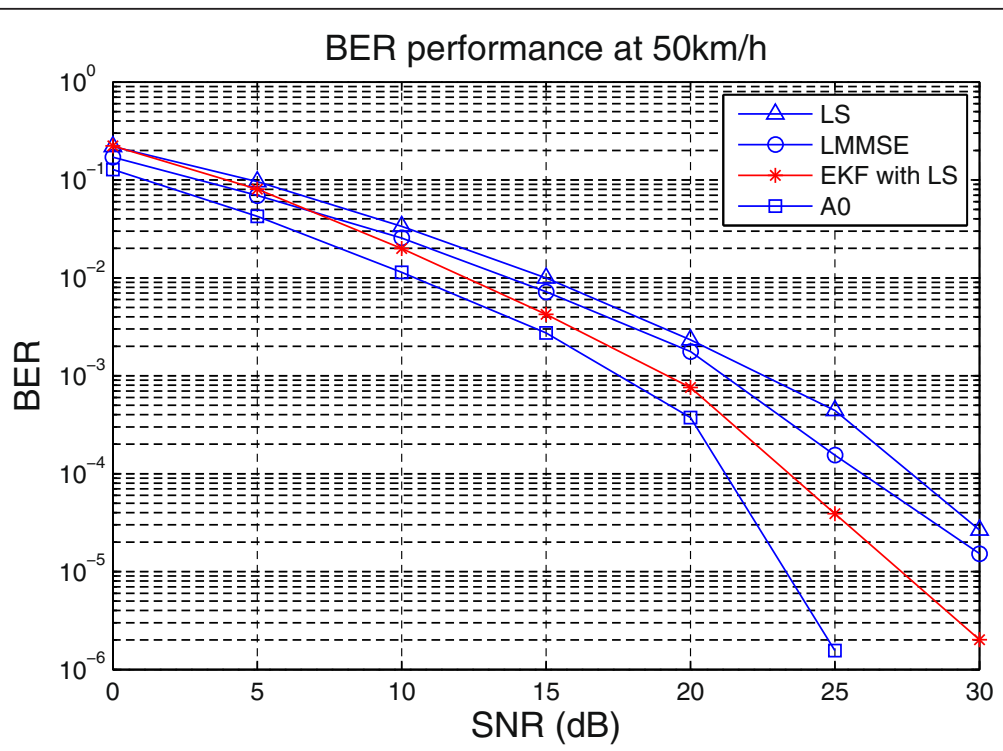

Figure 6 BER performance comparison at $50 \mathrm{~km} / \mathrm{h}$ (AO denotes a perfect channel estimation algorithm where the actual CFR is known to the receiver). Blue solid line with triangle: the BER performance of LS method. Red solid line with star: the BER performance of the proposed EKF method. Blue solid line with circle: the BER performance of LMMSE method. Blue solid line with square: the BER performance of the perfect channel estimation method. The perfect channel estimation means that the actual CFR is known to the receiver. 


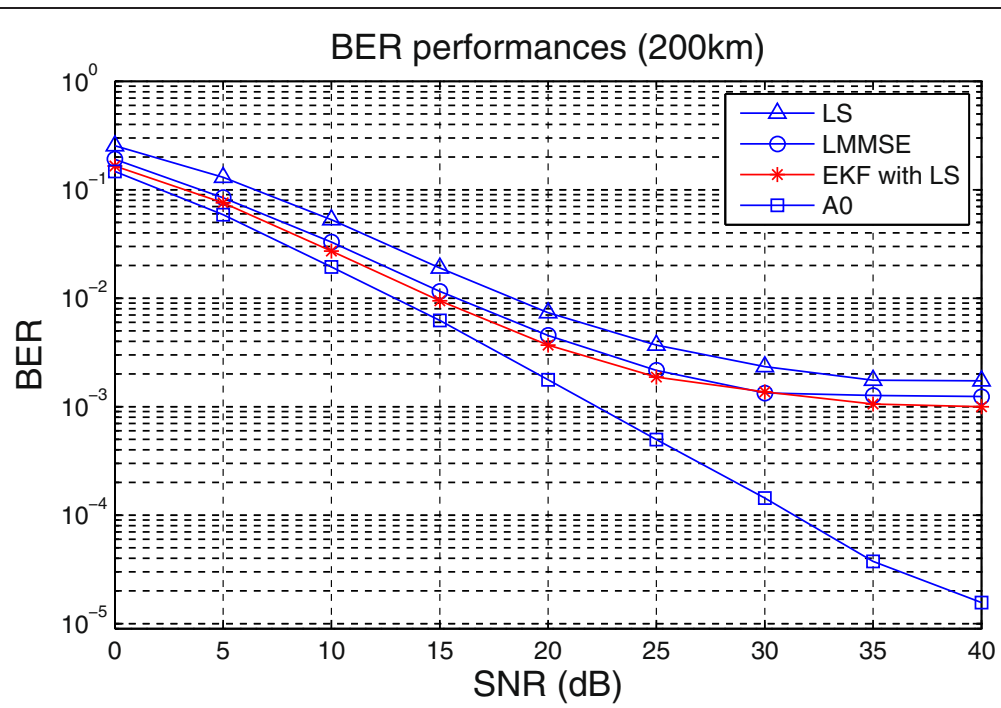

Figure 7 BER performance comparison at $200 \mathrm{~km} / \mathrm{h}$ (AO denotes a perfect channel estimation algorithm where the actual CFR is known to the receiver). Blue solid line with triangle: the BER performance of LS method.Red solid line with star: the BER performance of the proposed EKF method. Blue solid line with circle: the BER performance of the LMMSE method. Blue solid line with square: the BER performance of the perfect channel estimation method. The perfect channel estimation means that the actual CFR is known to the receiver.

the popular (LMMSE) algorithm [5] is also employed. Note that since the proposed EKF method is based on the LS estimation, we explicitly denote it by 'EKF with LS' in the legends of these figures. As a performance benchmark, the BER performances of a perfect channel estimation algorithm (denoted by A0 in Figures 6, 7 and 8) are also depicted, where the perfect channel estimation refers to the actual CFR being known by the receiver in advance.

As expected, A0 gives the best performance among all of the three methods, since it has the perfect CFR. The BER performance of A0 can be regarded as the
BER's lower bound. Obviously, the LS method has the poorest BER performance in all these three scenarios and the LMMSE is able to improve the BER performance. It can be seen that BERs of the proposed Kalman interpolation filter fall between the LMMSE's performances and the performances of perfect channel, although the EKF shows a slightly higher BER than LMMSE in low SNRs (i.e. 0 and $5 \mathrm{~dB}$ ). It is worth noting that the EKF is always better than the LS method. This is to be expected since the concept behind the observation equation in the proposed EKF method is the

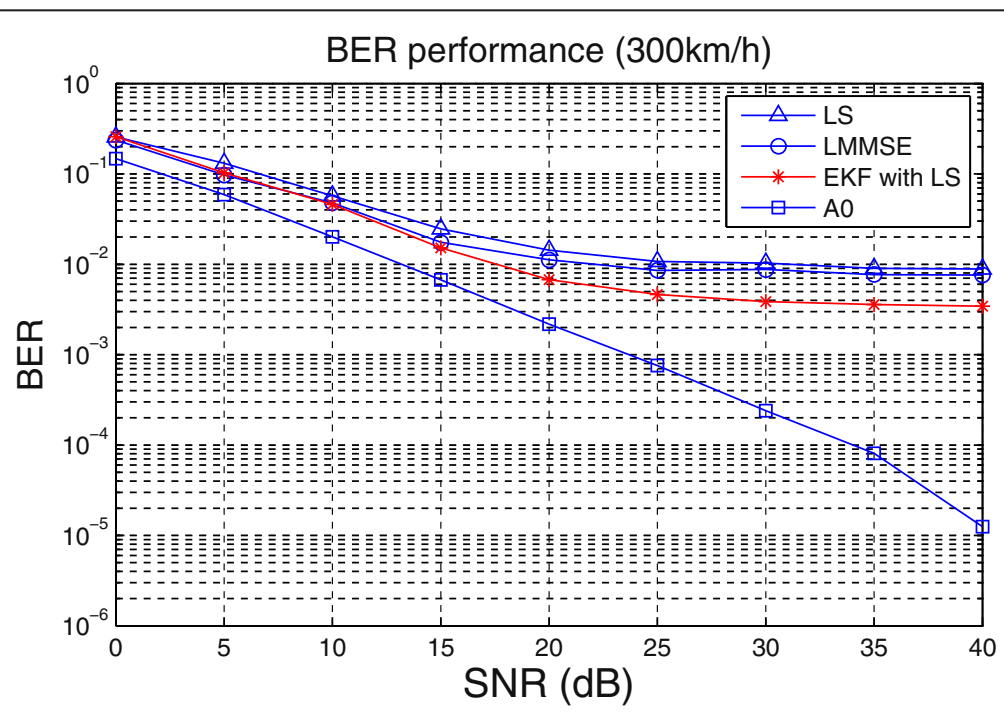

Figure 8 BER performance comparison at $300 \mathrm{~km} / \mathrm{h}$ (AO denotes a perfect channel estimation algorithm where the actual CFR is known to the receiver). 
same as the LS method, where it assumes the CFRs at adjacent pilot subcarriers are independent. Nevertheless, compared to the LS estimation, the proposed Kalman interpolation filter shows a significant improvement. This is particularly obvious at high SNRs and high-speed environment. As seen in Figure 7, when using the proposed EKF instead of the LS estimator, a gain in SNR up to $8 \mathrm{~dB}$ can be obtained for certain BERs (e.g. 0.002) at high-speed application. The average SNR gain is about $3-5 \mathrm{~dB}$.

\section{Conclusions}

This article focuses on channel estimation and interpolation for a time-varying multipath fading channel in 3GPP LTE downlink. The time-varying radio channel is modelled as an AR process represented in state space form and an EKF is developed for the purpose of both channel estimation at pilot symbols and interpolation at data symbols. The time-varying channel estimation is a joint state and parameter estimation problem, where both the channel taps and AR parameters need to be estimated simultaneously to achieve an accurate channel estimate. We convert the state model into an augmented system and a corresponding EKF is proposed. Furthermore, the interpolation channel estimate at data symbols are also integrated into the EKF and the proposed Kalman interpolation filter shows a good performance of estimating a time-varying channel in the 3GPP LTE downlink.

\section{Appendix}

Applying the first-order Taylor approximation to the nonlinear state transition function $f\left(\mathbf{z}_{n}\right)$ around $\hat{z}_{n \mid n}$ in Equation (17), the state equation (16) becomes

$$
\mathbf{z}_{n} \approx \mathbf{F}_{n} \mathbf{z}_{n}+\mu_{n}+u_{n}
$$

where $\mu_{n}$ is the linearization error $\mu_{n}=f\left(\hat{\mathbf{z}}_{n \mid n}\right)-\mathbf{F}_{n} \hat{\mathbf{z}}_{n \mid n}$ and

$$
\begin{aligned}
\mathbf{F}_{n} & \triangleq\left(\frac{\partial f\left(\mathbf{z}_{n}\right)}{\partial_{z_{n}}}\right)_{z_{n}=\hat{z}_{n \mid n}}=\left(\begin{array}{c}
\frac{\partial \mathbf{a}_{n}}{\partial \mathbf{z}_{n}} \\
\frac{\partial \mathbf{A}\left(\mathbf{a}_{n}\right) h_{n}}{\partial z_{n}^{T}}
\end{array}\right)_{z_{n}=\hat{z}_{n \mid n}} \\
& =\left(\begin{array}{c}
\frac{\partial \mathbf{a}_{n}}{\partial z_{n}^{T}} \\
\frac{\partial \mathbf{A}\left(\mathbf{a}_{n}\right)}{\partial z_{n}^{T}} \mathbf{h}_{n}+\mathbf{A}\left(\mathbf{a}_{n}\right) \frac{\partial \mathbf{h}_{n}}{\partial \mathbf{z}_{n}^{T}}
\end{array}\right)_{z_{n}=\hat{z}_{n \mid n}} \\
& =\left(\begin{array}{cc}
\mathbf{I}_{N_{A}} & 0 \\
\frac{\partial \mathbf{A}\left(\mathbf{a}_{n}\right)}{\partial \mathbf{a}_{n}^{T}} h_{n} & \mathbf{A}\left(\mathbf{a}_{n}\right)
\end{array}\right)_{z_{n}^{T}=\hat{z}_{n \mid n}}
\end{aligned}
$$

Here, we assume that $\mathbf{A}\left(\mathbf{a}_{k}\right)$ is independent of $\mathbf{h}_{n}$. Recalling the definitions of $\mathbf{a}_{k}$ and $\mathbf{A}\left(\mathbf{a}_{k}\right)$, it is easy to verify that $\frac{\partial A\left(a_{n}^{T}\right)}{\partial a_{n}} h_{n}$ is an $N_{p} \times N_{A}$ block-diagonal matrix $H_{n}$

$$
H_{n}=\mathcal{S}\left(I_{N_{p}} \otimes h_{n}^{T}\right)
$$

where $\otimes$ denotes the Kronecker product and the operator $\mathcal{S}(H)$ removes these known-columns of $I_{N_{p}} \otimes h_{n}^{T}$. The known column is the $(i-1) N_{p}+j$ th column when the $i$ th-row-jth-column entry $A_{i, j}$ of $\mathbf{A}$ is known. Hence, substituting $z_{n}=\hat{z}_{n \mid n}$ into Equation (26), we have

$$
F_{n}=\left[\begin{array}{cc}
I_{N_{A}} & 0 \\
\hat{H}_{n \mid n} & \hat{A}_{n \mid n}
\end{array}\right]
$$

And the linear state space model approximating the AR model (12) is

$$
\left\{\begin{array}{l}
\mathbf{z}_{n+1}=\mathbf{F}_{n} \mathbf{z}_{n}+\mathbf{u}_{n} \\
\mathbf{y}_{n}=\left[\begin{array}{ll}
0 & \mathbf{X}_{n}
\end{array}\right] \mathbf{z}_{n}+\mathbf{w}_{n}
\end{array}\right.
$$

\section{Abbreviations}

3GPP: The 3rd Generation Partnership Project (3GPP); AR: autoregressive; CIR: channel impulse response; DFT: discrete Fourier transform;

EKF: extended Kalman filter; LMMSE: linear minimum mean square error; LS: least square; LTE: long-term evolution; OFDM: orthogonal frequencydivision multiplexing; PSAM: pilot symbol-aided modulation;

QPSK: quadrature phase-shift keying; SNR: signal-to-noise ratio.

\section{Competing interests}

The authors declare that they have no competing interests.

\section{Acknowledgement}

This study was supported by the EPSRC UK-China Science Bridges: R\&D on 4 G Wireless Mobile Communications under grant EP/G042713/1.

\section{Author details}

${ }^{1}$ Department of Electronic \& Electrical Engineering, University College London, Torrington Place, London WC1E 7JE, UK. ${ }^{2}$ Graduate University of Chinese Academy of Sciences, Beijing 100049, China. ${ }^{3}$ Shanghai Research Center for Wireless Communications (WiCO), Shanghai 200050, China.

${ }^{4}$ Shanghai Institute of Microsystem and Information Technology (SIMIT), Shanghai 200050, China.

Received: 23 August 2011 Accepted: 11 June 2012 Published: 25 July 2012

\section{References}

1. B. Karakaya, An adaptive channel interpolator based on Kalman filter for LTE uplink in high Doppler spread environments. EURASIP J. Wirel. Commun. Netw. 2009, 1-10 (2009)

2. J.K. Cavers, An analysis of pilot symbol assisted modulation for Rayleigh fading channels. IEEE Trans. Veh. Technol. 40(4), 686-693 (1991)

3. F. Tufvesson, T. Maseng, Pilot assisted channel estimation for OFDM in mobile cellular systems. in Proc. of IEEE Vehicular Technology Conference'97 3, 1639-1643 (1997). Phoenix, Az

4. M.H. Hsieh, C.H. Wei, Channel estimation for OFDM systems based on comb-type pilot arrangement in frequency selective fading channels. IEEE Trans. Consum. Electron. 44(1), 217-225 (1998)

5. J. Beek, O. Edfors, M. Sandell, S. Wilson, P. Borjesson, On channel estimation in OFDM systems. in Proc. of IEEE Vehicular Technology Conference'95 2, 815-819 (1995). Chicago, II 
6. P. Hoecher, S. Kaiser, P. Robertson, Pilot-symbol-aided channel estimation in time and frequency. in Proc. of IEEE Global Telecommunications Conference'97 Communication Theory Mini-Conference 3, 90-96 (1997). Phoenix, Az

7. W.H. Chin, D.B. Ward, A.G. Constantinides, An algorithm for exploiting channel time selectivity in pilot-aided MIMO systems. IET Commun. 1(6), 1267-1273 (2007)

8. O. Edfors, J. van de Beek, M. Sandell, S.K. Wilson, P.O. Borjesson, Analysis of DFT-based channel estimators for OFDM. Int. J. Wirel. Personal Commun. 12(1), 55-70 (2000)

9. Steepest Ascent Ltd, Improving throughput performance in LTE by channel estimation noise averaging, The LTE-Advanced Guide (, 2010). Online, http:// www.steepestascent.com/content/mediaassets/pdf/products/ LTE_Portal_Article_May_2010.pdf

10. J. Hou, J. Liu, A novel channel estimation algorithm for 3GPP LTE downlink system using joint time-frequency two-dimensional iterative Wiener filter. in Proc. of 12th IEEE Int. Conf. on Communication Technology (ICCT) 1, 289-292 (2010). Nanjing, China

11. R. Iltis, Joint estimation of PN code delay and multipath using the extended Kalman filter. IEEE Trans. Commun. 88(10), 1677-1683 (1990)

12. K.-Y. Han, S.-W. Lee, J.-S. Lim, K.-M. Sung, Channel estimation for OFDM with fast fading channels by modified Kalman filter. IEEE Trans. Consum. Electron. 50(2), 443-449 (2004)

13. M. Huang, X. Chen, L. Xiao, S. Zhou, J. Wang, Kalman-filter-based channel estimation for orthogonal frequency-division multiplexing systems in timevarying channels. IET Commun. 1(1), 759-801 (2007)

14. S.K. Mitra, Digital Signal Processing: A Computer-Based Approach, 2nd edn. (McGraw-Hill//rwin, Boston, MA, 2001)

15. L.M. Davis, I. Collings, R. Evans, Coupled estimators for equalization of fastfading mobile channels. IEEE Trans. Commun. 46(10), 1262-1265 (1998)

16. W. Li, Estimation and tracking of rapidly time-varying broadband acoustic communication channels, Ph.D. dissertation, Massachusetts Institute of Technology \& Woods Hole Oceanographic Institution (, 2006)

17. 3GPP, Technical specification group radio access network, Deployment aspects (release 10), 3GPP TR 25.943V10.0.0," 3GPP, Technical Report, (April 2011). Online, http://www.3gpp.org/ftp/specs/html-info/25943.htm

doi:10.1186/1687-1499-2012-232

Cite this article as: Dai et al:: Kalman interpolation filter for channel estimation of LTE downlink in high-mobility environments. EURASIP Journal on Wireless Communications and Networking 2012 2012:232.

\section{Submit your manuscript to a SpringerOpen ${ }^{\odot}$ journal and benefit from:}

- Convenient online submission

- Rigorous peer review

- Immediate publication on acceptance

- Open access: articles freely available online

- High visibility within the field

- Retaining the copyright to your article

Submit your next manuscript at $\gg$ springeropen.com 